\title{
A terminator of floral stem cells
}

\author{
Feng Ming ${ }^{1}$ and Hong $M^{1,2,3,4,5}$ \\ ${ }^{1}$ State Key Laboratory of Genetic Engineering and Institute of Plant Biology, School of Life Sciences, Fudan University, Shanghai \\ 200433 China; ${ }^{2}$ The Center for Evolutionary Biology, the School of Life Sciences, Fudan University, Shanghai 200433 China; \\ ${ }^{3}$ The Institutes of Biological Sciences, Fudan University, Shanghai 200032 China; ${ }^{4}$ Department of Biology and the Huck \\ Institutes of the Life Sciences, Institute of Molecular Evolutionary Genetics, the Pennsylvania State University, University Park, \\ Pennsylvania 18602, USA
}

\begin{abstract}
Normal flower development requires the termination of stem cell activities in the floral meristem. The floral regulator AGAMOUS $(A G)$ is necessary for this termination and represses the expression of the stem cell determinant WUSCHEL (WUS), but the repression mechanism was not clear. A recent study by Sun and colleagues (pp. 1791-1804) in this issue of Genes \& Development has identified a direct target of AG, KNUCKLES (KNU), which encodes a transcriptional repressor of WUS, providing a key missing link in floral meristem determinacy.
\end{abstract}

Post-embryonic organ formation in flowering plants requires the activities of stem cells in the shoot, floral, and root meristems (Weigel and Jurgens 2002; Laux 2003). To produce multiple organs, the meristems need to maintain stem cells for sufficient periods of time. In Arabidopsis, this maintenance of stem cells requires the function of WUSCHEL (WUS), which was shown to be necessary and sufficient to promote stem cell activities in the meristems (Mayer et al. 1998). Another Arabidopsis regulator of meristem activity is SHOOTMERISTEMLESS (STM). STM and WUS function independently, though an increase in WUS function can partly compensate for the loss of STM function (Lenhard et al. 2002). WUS specifies a subset of cells in the center of the meristem as stem cells, whereas STM acts to suppress differentiation throughout the meristem, thus allowing the daughter cells of stem cells to proliferate before they are incorporated into organs (Lenhard et al. 2002).

The floral meristem differs from others in being determinate, generating a constant or limited number of floral organs before ceasing its activity. In addition, the termination of stem cell activities in the floral meristem is coupled to the initiation of reproductive floral organs and the timely determinacy of the floral meristem is important for proper formation of reproductive organs, particularly the female organ carpel. In Arabidopsis, the

[Keywords: Arabidopsis thaliana; flower development; homeotic control; MADS protein; AGAMOUS; WUSCHEL; stem cell] Correspondence.

${ }^{5}$ E-MAIL hongma@fudan.edu.cn or hxm16@psu.edu; FAX (814) 863-1357. Article is online at http://www.genesdev.org/cgi/doi/10.1101/gad.1834409. floral homeotic gene AGAMOUS $(A G)$ is required for both floral meristem determinacy and reproductive organ identities (Bowman et al. 1989, 1991; Yanofsky et al. 1990; Coen and Meyerowitz 1991; Mizukami and Ma 1995). $A G$ is a key gene for the $C$ function of the $\mathrm{ABC}$ model and a member of the MADS-box gene family (Yanofsky et al. 1990; Bowman et al. 1991; Coen and Meyerowitz 1991). In ag mutants, the floral meristem is active for a much longer period than normal, generating many more floral organs than the wild type (Bowman et al. 1989, 1991; Yanofsky et al. 1990). Similarly, reduction of $A G$ function using sense or antisense transgenes causes extended floral meristem activities (Mizukami and Ma 1995; Mizukami et al. 1996).

Thus, proper stem cell activity in the floral meristem is controlled by the balance between the positive regulator WUS and the negative regulator $A G$. This is achieved through a positive-negative feedback loop (Fig. 1A). WUS is expressed in the early floral meristem and activates $A G$ expression at floral stage 3 (as defined by Smyth et al. 1990). AG expression is reduced in the wus mutant, whereas ectopic WUS expression prolongs stem cell proliferation and also causes ectopic stamen and carpel development in an AG-dependent way (Lenhard et al. 2001; Lohmann et al. 2001). DNA-binding studies suggest that WUS cooperates with another positive regulator of floral meristem, LEAFY (LFY), to activate $A G$ expression (Lohmann et al. 2001). On the other hand, WUS expression decreases when $A G$ expression is activated and disappears by the time carpel primordia are initiated at stage 6 (Mayer et al. 1998). WUS is required for the floral meristem indeterminacy in the $a g-1$ mutant because the flowers of the wus-1 ag-1 double mutant appear indistinguishable from those of wus-1 (Laux et al. 1996), supporting the hypothesis that AG negatively regulates WUS expression. Indeed, WUS remains active in the center of the indeterminate floral meristem of the $a g-1$ mutant through the life of the flower (Lenhard et al. 2001; Lohmann et al. 2001), indicating that $A G$ is required for the cessation of WUS expression at stage 6.

Clearly, the precise duration of stem cell activity in the floral meristem is important for normal development. Terminating the stem cells too early would mean insufficient cells for floral organ formation, whereas overly extended 


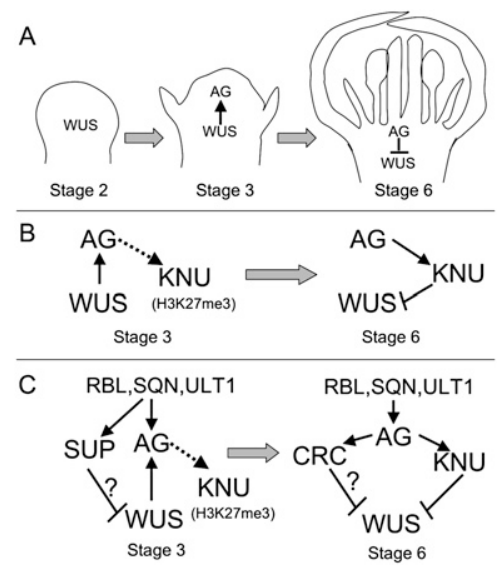

Figure 1. Genes controlling stem cell activities in the floral meristem. (A) A regulatory loop between WUS and AG. WUS is expressed at floral stage 2 and then activates (arrow) $A G$ expression at stage 3 . By stage $6, A G$ has repressed (line with a bar) WUS. (B) Interactions between $A G, K N U$, and WUS. At stage 3, AG binds to the $K N U$ promoter (dotted arrow), but H3K27 at $\mathrm{KNU}$ is trimethylated and KNU has no effect on WUS. At stage 6, the $\mathrm{H} 3 \mathrm{~K} 27$ trimethylation is removed and $K N U$ is expressed, causing WUS repression. (C) Regulation of stem cell activity by AG, KNU, WUS, and other factors. At stage 3, RBL, SQN, and $U L T 1$ positively regulate $A G$ and $S U P$ expression, the latter of which might also repress WUS via an unknown mechanism. At stage 6, AG can activate the expression of $C R C$, which also represses WUS.

stem cell activity would result in abnormally large numbers of floral organs. Furthermore, because of the coupling of floral meristem termination with the formation of the carpels, the female reproductive organs, extended meristem activity is also linked to defects in carpel development. Thus, the above-mentioned positive-negative feedback loop must have a timing component: WUS activates $A G$ expression first at stage 3 , and AG represses WUS expression subsequently at stage 6 . How is differential timing of the two aspects of the regulatory loop achieved? In particular, what is the mechanism for the delayed repression of WUS by AG?

Sun et al. (2009) have now obtained strong support from a series of genetic and molecular experiments for another gene, KNUCKLES $(K N U)$, that serves to mediate the negative regulation of WUS by AG, and provide evidence for the timing mechanism of the termination of WUS expression. KNU was discovered as a positive regulator of floral meristem determinacy; unlike the normal flower, which produces carpels as the fourth and innermost whorl of organs, knu mutant flowers have extra stamens and carpels that form interior to the fourth-whorl carpels (Payne et al. 2004). In addition, the KNU protein contains a zinc finger and a putative transcriptional repression motif (Payne et al. 2004), suggesting that it might be a repressor of WUS. Therefore, Sun et al. (2009) hypothesized that KNU might act between $A G$ and $W U S$ to mediate the repression of WUS by AG.

To investigate how $A G$ regulates floral meristem determinacy and the role of $K N U$ in this process, Sun et al.
(2009) performed a series of genetic and molecular experiments. First, they ascertained the time at which $A G$ is needed for normal determinacy, using an elegant experiment with inducible AG activity. It was shown previously that AG activity can be controlled by fusing it with a mammalian steroid hormone receptor (glucocorticoid receptor [GR]) that can be activated by an artificial hormone, dexamethosone (dex) (Ito et al. 2004; Gomez-Mena et al. 2005). By inducing AG activity at different floral stages in transgenic plants with inducible AG activity in an ag mutant background, Sun et al. (2009) showed that AG activity at stage 3 , but not stage 4 , was able to confer full determinacy, indicating that the normal onset of $A G$ function at stage 3 is required for floral meristem determinacy.

Next, Sun et al. (2009) tested the role of KNU by performing genetic experiments with various combinations of $a g$, wus, and knu mutations. They found that ag knu double mutants had similar phenotypes to those of $a g$ mutants, consistent with the idea that $A G$ and $K N U$ act in the same genetic pathway and that $A G$ is a positive regulator of $K N U$. In addition, the knu wus double mutant appeared the same as the wus mutant, indicating that WUS is downstream from KNU. Furthermore, when $K N U$ was expressed using an AG-independent promoter (35S), KNU was able to rescue the indeterminate phenotype of the ag-1 mutant, suggesting that KNU functions downstream from $A G$. On the other hand, AG-independent $K N U$ expression caused wus-like floral phenotypes, supporting the hypothesis that KNU represses WUS expression. These experiments with mutants and transgenic plants provide key genetic evidence that KNU acts downstream from $A G$ to repress WUS (Fig. 1B).

To examine the relationship between $A G, K N U$, and WUS further at the level of mRNA expression, Sun et al. (2009) used a transgenic plant system that allows flower development to be induced and found that $K N U$ expression begins $\sim 2 \mathrm{~d}$ after the start of $A G$ expression, whereas WUS expression is repressed starting at $\sim 2 \mathrm{~d}$ after $A G$ expression. More directly, they showed that induction of AG activity using the $A G-G R$ fusion system is followed by $K N U$ expression $\sim 2 \mathrm{~d}$ later. In contrast, knu mutants exhibited extended WUS expression, indicating that KNU is required for the termination of WUS expression in the floral meristem. Further support for the negative regulation of WUS by KNU came from transgenic plants with strong inducible KNU activity, which resulted in premature termination of meristem activity similar to that found in wus mutants. Sun et al. (2009) also determined that the repression motif at the $\mathrm{C}$-terminal region of the $\mathrm{KNU}$ protein was required for termination of WUS expression, suggesting that KNU acts as a transcriptional repressor.

The delay in $K N U$ expression in comparison with $A G$ expression suggests that the activation of $K N U$ expression might be a key to the timing of stem cell termination in the floral meristem, thereby allowing the needed amount of stem activity for floral organ generation. To examine this temporal relationship between $A G$ and $K N U$ further, Sun et al. (2009) combined the inducible AG /using the AG-GR fusion) with a KNU-GUS reporter gene and found that $1 \mathrm{~d}$ after AG induction, GUS expression was 
not detected, but $2 \mathrm{~d}$ after AG induction, strong GUS expressed was detected. As a positive control, another AG target gene, SPL, was activated immediately after AG induction. Although it is not known how KNU expression is delayed compared with AG induction, the delayed $K N U$ expression is likely a key to allowing WUS to be expressed long enough for sufficient floral stem cell activity to generate floral organs before the termination of the floral meristem.

The next question Sun et al. (2009) addressed was whether $K N U$ is a direct target of transcriptional regulation by AG. Sun et al. (2009) noted that the putative KNU promoter region does not contain a perfect AG target site, as defined by earlier in vitro studies (Huang et al. 1993; Shiraishi et al. 1993); nevertheless, there are three partial sites of AG-binding consensus sequences that were found previously to bind to AG weakly (Ito et al. 1997). To test whether AG binds to the $K N U$ promoter region in vivo, Sun et al. (2009) performed chromatin immunoprecipitation (ChIP) experiments. They found that the KNU promoter region containing the partial AG-binding sites was enriched after $1 \mathrm{~d}$ of the induction of AG activity, and this enrichment was further strengthened 2 and $3 \mathrm{~d}$ after induction. Furthermore, when the partial AG-binding sites were mutated in a fusion of the $K N U$ promoter to the GUS reporter gene, GUS expression was not detected in $88 \%$ of the transgenic plants, in contrast to GUS expression in $80 \%$ of the transgenic plants carrying a fusion with the wild-type $K N U$ promoter. These results support the hypotheses that AG binds directly to the $K N U$ promoter and that AG binding activates $K N U$ transcription.

At the same time, it was puzzling why AG binding was observed by ChIP $1 \mathrm{~d}$ after the induction of AG function, but $K N U$ expression was not detectable until $2 \mathrm{~d}$ after the induction. To probe further into the mechanism of the activation of KNU expression, Sun et al. (2009) examined the status of transcription-repressive marker histone $\mathrm{H} 3$ Lys 27 trimethylation (H3K27me3). They found that the level of H3K27me3 was high near the KNU transcriptional start and in the coding region; moreover, the H3K27me3 level at KNU was reduced $2 \mathrm{~d}$, but not $1 \mathrm{~d}$, after flower development was induced, in an AG-dependent manner. So, the timing of the reduction of the H3K27me3 level is consistent with that of KNU expression. Also, Sun et al. (2009) found that a deletion of the KNU region with heavy $\mathrm{H} 3 \mathrm{~K} 27 \mathrm{me} 3$ resulted in elevated expression of a $K N U-G U S$ reporter gene.

Is the H3K27me3 level, or some other cis elements in the deleted $K N U$ region, relevant to $K N U$ regulation? Sun et al. (2009) addressed this question by testing for KNU expression in mutants that are defective in genes encoding polycomb group proteins, which maintain H3K27me3 levels (Katz et al. 2004; Schubert et al. 2006). Indeed, $K N U$ was expressed ectopically in leaves of these mutants. Another test was to see whether another factor associated with $\mathrm{H} 3 \mathrm{~K} 27 \mathrm{me} 3$, the epigenetic repressor TFL2/LHP1, was involved in repressing KNU expression. In the tfl2 mutant, $K N U$ was overexpressed in the flower and ectopically expressed in the inflorescence stem. Therefore, defects in maintaining H3K27me3 levels could de- repress $K N U$ expression, suggesting that relatively high levels of H3K27me3 at the KNU locus might be responsible for the repression of $K N U$ expression, although the formal possibility that the epigenetic regulators polycomb group proteins and TFL2 might affect $K N U$ expression indirectly could not be ruled out.

Whereas Sun et al. (2009) convincingly showed that AG likely activates $K N U$ transcription by binding directly to the $K N U$ promoter and that $K N U$ expression is delayed relative to the onset of AG function, probably by repressive chromatin as marked by $\mathrm{H} 3 \mathrm{~K} 27 \mathrm{me} 3$, several questions remain. First, it is not known whether and how AG acts to counter the repressive chromatin. Second, the difference for AG function between $1 \mathrm{~d}$ and 2 $\mathrm{d}$ after AG is induced is not known. In other words, why was AG able to counter the effect of repressive chromatin $2 \mathrm{~d}$, but not $1 \mathrm{~d}$, after AG induction? Sun et al. (2009) proposed that the repressive marker is removed in an AGdependent manner and suggested that the period of $2 \mathrm{~d}$ might be required because of a need for active cell division. However, AG is not a histone demethylase. Could AG recruit a histone demethylase to the KNU promoter region? Is the removal of histone methylation achieved passively by preventing methylation following DNA replication during the cell cycle?

A second area that requires further investigation concerns the mechanism by which KNU represses WUS expression. Although Sun et al. (2009) showed that the KNU repression motif is required for the termination of WUS expression, it is possible that KNU represses an activator of WUS expression, thereby negatively regulating WUS expression in an indirect fashion. Sun et al. (2009) indicated that they performed a ChIP experiment for KNU binding to the WUS promoter, but the result was not conclusive. Perhaps more importantly, KNU has not been shown to be, or to associate with, a DNA-binding protein, although it has a $\mathrm{C} 2 \mathrm{H} 2$-type zinc finger (Payne et al. 2004). Nevertheless, the observation by Sun et al. (2009) that the activity of a KNU-GR fusion protein can be induced by the artificial hormone dex suggests that $\mathrm{KNU}$ is a transcriptional regulator. Future experiments are needed to determine whether KNU is a DNA-binding protein and, if so, what the binding sequence for $\mathrm{KNU}$ is.

Yet another puzzle is the relationship between $A G$, $K N U$, and other genes that also play a role in regulating floral meristem determinacy (Fig. 1C). One such additional regulator is the $\mathrm{C} 2 \mathrm{H} 2$ zinc finger protein SUPERMAN (SUP), which has been shown to repress stem cell proliferation at floral stages 3 and 4 (Bowman et al. 1992; Sakai et al. 1995). Genetic studies indicated that $A G$ and SUP control floral meristem determinacy through independent pathways (Schultz et al. 1991; Bowman et al. 1992). SUP is also involved in the termination of WUS expression (Prunet et al. 2008). What is the relationship between the two $\mathrm{C} 2 \mathrm{H} 2$ zinc finger proteins $\mathrm{KNU}$ and SUP? Another contributor to the floral determinacy is CRABS CLAW (CRC), which also negatively regulates stem cell proliferation (Bowman and Smyth 1999) and might act downstream from $A G$ and two other floral organ identity genes-APETALA3 and PISTILLATA (Lee et al. 2005). 
However, the relationship between $C R C$ and $K N U$ is unclear, and the interactions between $A G, S U P$, and $C R C$ at the molecular level are yet to be understood. Recent studies have uncovered other positive regulators of floral meristem determinacy-REBELOTE (RBL), SQUINT $(S Q N)$, and ULTRAPETALA1 (ULT)-that function upstream of $A G$ and SUP (Fig. 1C; Carles et al. 2004; Prunet et al. 2008). The proteins encoded by these genes have various predicted activities, suggesting that they act with different mechanisms. With rapid progress fueled by new technologies, future investigations will likely yield new insights regarding the control of stem cell activities in the floral meristem, ever enriching us in this fertile ground of plant development.

\section{Acknowledgments}

F.M. was supported by a grant from the Ministry of Agriculture of China (2008ZX08009-001), the Program for the Youth Science and Technology Phosphor Foundation of Shanghai (08QH14003), and the Science and Technology Foundation of Shanghai (083391910400). H.M. was supported by the U.S. Department of Energy (DE-FG02-02ER15332) and funds from Rijk Zwaan, Fudan University, and the Pennsylvania State University.

\section{References}

Bowman JL, Smyth DR. 1999. CRABS CLAW, a gene that regulates carpel and nectary development in Arabidopsis, encodes a novel protein with zinc finger and helix-loop-helix domains. Development 126: 2387-2396.

Bowman JL, Smyth DR, Meyerowitz EM. 1989. Genes directing flower development in Arabidopsis. Plant Cell 1: 37-52.

Bowman JL, Smyth DR, Meyerowitz EM. 1991. Genetic interactions among floral homeotic genes of Arabidopsis. Development 112: 1-20.

Bowman JL, Sakai H, Jack T, Weigel D, Mayer U, Meyerowitz EM. 1992. SUPERMAN, a regulator of floral homeotic genes in Arabidopsis. Development 114: 599-615.

Carles CC, Lertpiriyapong K, Reville K, Fletcher JC. 2004. The ULTRAPETALA1 gene functions early in Arabidopsis development to restrict shoot apical meristem activity and acts through WUSCHEL to regulate floral meirstem determinacy. Genetics 167: 1893-1903.

Coen ES, Meyerowitz EM. 1991. The war of the whorls: Genetic interactions controlling flower development. Nature 353: 31-37.

Gomez-Mena C, de Folter S, Costa MMR, Angenent GC, Sablowski R. 2005. Transcriptional program controlled by the floral homeotic gene AGAMOUS during early organogenesis. Development 132: 429-438.

Huang H, Mizukami Y, Hu Y, Ma H. 1993. Isolation and characterization of the binding sequences for the product of the Arabidopsis floral homeotic gene AGAMOUS. Nucleic Acids Res 21: 4769-4776.

Ito T, Takahashi N, Shimura Y, Okada K. 1997. A serine/ threonine protein kinase gene isolated by an in vivo binding procedure using the Arabidopsis floral homeotic gene product, AGAMOUS. Plant Cell Physiol 38: 248-258.

Ito $\mathrm{T}$, Wellmer $\mathrm{F}, \mathrm{Yu} \mathrm{H}$, Das $\mathrm{P}$, Ito $\mathrm{N}$, Alves-Ferreira $\mathrm{M}$, Riechmann JL, Meyerowitz EM. 2004. The homeotic protein AGAMOUS controls microsporogenesis by regulation of SPOROCYTELESS. Nature 430: 356-360.

Katz A, Oliva M, Mosquna A, Hakim O, Ohad N. 2004. FIE and CURLY LEAF polycomb proteins interact in the regulation of homeobox gene expression during sporophyte development. Plant J 37: 707-719.

Laux T. 2003. The stem cell concept in plants: A matter of debate. Cell 113: 281-283.

Laux T, Mayer KFX, Berger J, Jurgens G. 1996. The WUSCHEL gene is required for shoot and floral meristem integrityin Arabidopsis. Development 122: 87-96.

Lee JY, Baum SF, Alvarez J, Patel A, Chitwood DH, Bowman JL. 2005. Activation of CRABS CLAW in the nectaries and carpels of Arabidopsis. Plant Cell 17: 25-36.

Lenhard M, Bohnert A, Jurgens G, Laux T. 2001. Termination of stem cell maintenance in Arabidopsis floral meristems by interactions between WUSCHEL and AGAMOUS. Cell 105: 805-814.

Lenhard M, Jürgens G, Laux T. 2002. The WUSCHEL and SHOOTMERISTEMLESS genes fulfill complementary roles in Arabidopsis shoot meristem regulation. Development 129: 3195-3206.

Lohmann J, Huong R, Hobe M, Busch M, Parcy F, Simon R, Weigel D. 2001. A molecular link between stem cell regulation and floral patterning in Arabidopsis. Cell 105: 793-803.

Mayer KFX, Schoof H, Haecker A, Lenhard M, Jurgens G, Laux T. 1998. Role of WUSCHEL in regulating stem cell fate in the Arabidopsis shoot meristem. Cell 95: 805-815.

Mizukami Y, Ma H. 1995. Separation of $A G$ function in floral meristem determinacy from that in reproductive organ identity by expressing antisense AG RNA. Plant Mol Biol 28: 767-784.

Mizukami Y, Huang H, Tudor M, Hu Y, Ma H. 1996. Functional domains of the floral regulator AGAMOUS: Characterization of the DNA-binding domain and analysis of dominant negative mutations. Plant Cell 8: 831-845.

Payne T, Johnson SD, Koltunow AM. 2004. KNUCKLES (KNU) encodes a $\mathrm{C} 2 \mathrm{H} 2$ zinc-finger protein that regulates development of basal pattern elements of the Arabidopsis gynoecium. Development 131: 3737-3749.

Prunet N, Morel P, Thierry A, Eshed Y, Bowman J, Negrutiu I, Trehin C. 2008. REBELOTE, SQUINT, and ULTRAPETALA1 function redundantly in the temporal regulation of floral meristem termination in Arabidopsis thaliana. Plant Cell 20: $901-919$.

Sakai H, Medrano LJ, Meyerowitz EM. 1995. Role of SUPERMAN in maintaining Arabidopsis floral whorl boundaries. Nature 378: 199-203.

Schubert D, Primavesi L, Bishopp A, Roberts G, Doonan J, Jenuwein T, Goodrich J. 2006. Silencing by plant Polycombgroup genes requires dispersed trimethylation of histone $\mathrm{H} 3$ at lysine 27. EMBO I 25: 4638-4649.

Schultz EA, Pickett FB, Haughn GW. 1991. The FLO10 gene product regulates the expression domain of homeotic genes AP3 and PI in Arabidopsis flowers. Plant Cell 3: 1221-1237.

Shiraishi H, Okada K, Shimura Y. 1993. Nucleotide sequences recognized by the AGAMOUS MADS domain of Arabidopsis thaliana in vitro. Plant J 4: 385-398.

Smyth D, Bowman JL, Meyerowitz EM. 1990. Early flower development in Arabidopsis. Plant Cell 2: 755-767.

Sun B, Xu Y, Ng K-H, Ito T. 2009. A timing mechanism for stem cell maintenance and differentiation in the Arabidopsis floral meristem. Genes \& Dev (this issue). doi: 10.1101/gad.1800409.

Weigel D, Jurgens G. 2002. Stem cells that make stems. Nature 415: 751-754.

Yanofsky MF, Ma H, Bowman JL, Drews GN, Feldmann K, Meyerowitz EM. 1990. The protein encoded by the Arabidopsis homeotic gene agamous resembles transcription factors. Nature 346: 35-39. 


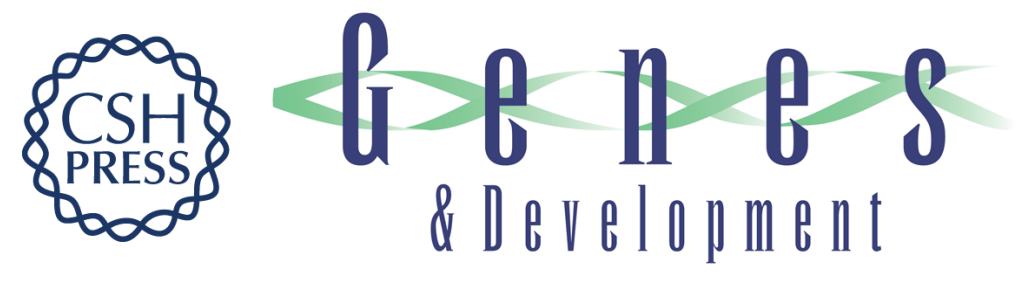

\section{A terminator of floral stem cells}

Feng Ming and Hong Ma

Genes Dev. 2009, 23:

Access the most recent version at doi:10.1101/gad.1834409

\section{Related Content A timing mechanism for stem cell maintenance and differentiation in the Arabidopsis floral meristem \\ Bo Sun, Yifeng Xu, Kian-Hong Ng, et al. \\ Genes Dev. August , 2009 23: 1791-1804 \\ References This article cites 30 articles, 15 of which can be accessed free at: \\ http://genesdev.cshlp.org/content/23/15/1705.full.html\#ref-list-1 \\ Articles cited in: \\ http://genesdev.cshlp.org/content/23/15/1705.full.html\#related-urls \\ License \\ Email Alerting \\ Service \\ Receive free email alerts when new articles cite this article - sign up in the box at the top right corner of the article or click here.}

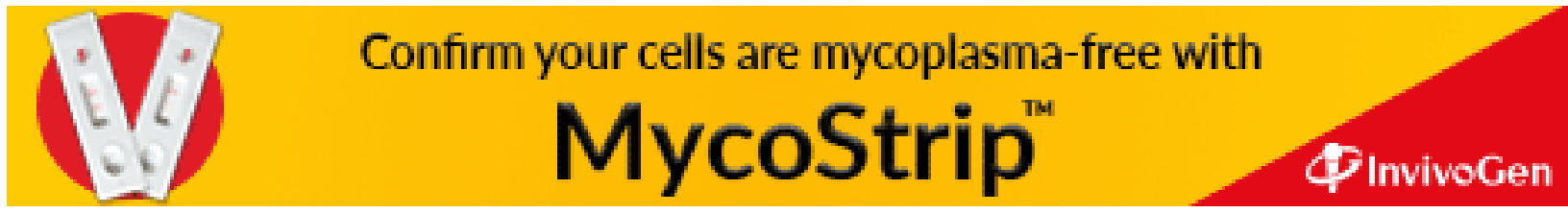

